\title{
Auroral pulsations and accompanying VLF emissions
}

\author{
V. R. Tagirov ${ }^{1}$, V. S. Ismagilov ${ }^{1}$, E. E. Titova ${ }^{1}$, V. A. Arinin $^{2}$, A. M. Perlikov ${ }^{1}$, J. Manninen ${ }^{3}$, T. Turunen ${ }^{3}$, \\ and K. Kaila ${ }^{4}$ \\ ${ }^{1}$ Polar Geophysical Institute, Apatity, Murmansk region, 184200, Russia \\ ${ }^{2}$ Russian Federal Nuclear Center, Sarov, Nizhni Novgorod region, 607190, Russia \\ ${ }^{3}$ Geophysical Observatory, FIN-99600, Sodankylä, Finland \\ ${ }^{4}$ University of Oulu, FIN-90570, Oulu, Finland
}

Received: 21 November 1997 / Revised: 13 May 1998 / Accepted: 3 June 1998

\begin{abstract}
Results of simultaneous TV observations of pulsating auroral patches and ELF-VLF-emissions in the morning sector carried out in Sodankylä (Finland) on February 15, 1991 are presented. Auroral pulsating activity was typical having pulsating patches with characteristic periods of about $7 \mathrm{~s}$. Narrow-band hiss emissions and chorus elements at intervals of $0.3-0.4 \mathrm{~s}$ formed the main ELF-VLF activity in the frequency range $1.0-2.5 \mathrm{kHz}$ at the same time. The analysis of auroral images with time resolution of $0.04 \mathrm{~s}$ allowed perfectly separate analysis of spatial and temporal variations in the auroral luminosity. Mutual correspondence between the behaviour of the luminous auroral patches and the appearance of ELF noise type hiss emissions and VLF chorus trains was found in two intervals chosen for analysis. While the hiss emissions were associated with the appearance of luminosity inside a limited area close to the zenith, the structured VLF emissions were accompanied by rapid motion of luminosity inside the area. The spatial dimension of the pulsating area was about $45-50 \mathrm{~km}$ and luminosity propagated inside it with velocity of about $10-12 \mathrm{kms}$. We discuss a new approach to explain the 5-15 s auroral pulsation based on the theory of flowing cyclotron maser and relaxation characteristics of ionosphere.
\end{abstract}

Key words. Magnetospheric physics (auroral phenomena; magnetosphere-ionosphere interactions) - Space plasma physics (wave-particle interactions).

\section{Introduction}

It is generally accepted that the whistler mode VLF waves play an important role in precipitation of

Correspondence to: V. R. Tagirov

E-mail: tagirov@pgi-ksc.murmansk.su energetic particles (Kennel, 1969; Inan et al., 1978) in connection with the auroral pulsations. Electrons close to the equatorial plane in the magnetosphere experience pitch angle scattering caused by VLF waves and this is followed by precipitation into the ionosphere. Rosenberg et al. (1971) presented an experimental evidence for such interactions. They found peak to peak correlation between discrete VLF emissions and spikes of X-rays with energies exceeding $30 \mathrm{keV}$ at balloon flight altitude near $L=4.2$.

There are different types of pulsating aurora observed during post-breakup period. The main types were defined by Yamamoto and Oguti (1982). Røyrvic and Davis (1977) determined the range of spatial dimensions of pulsating patches from 20 to $200 \mathrm{~km}$. Scourfield et al. (1972) studying a spatial coherency of pulsating aurora found that pulsations are coherent at the distances less than $30 \mathrm{~km}$. Tagirov et al. (1985) using statistical analysis of hundreds of pulsations on the basis of TV data found the period range of pulsations is from 5 to $40 \mathrm{~s}$ with the mean value $10 \mathrm{~s}$.

In this work we will deal with two types of pulsations. The first one is pure or stable pulsations which were defined as pulsations of luminosity in restricted area without any appreciable changes in sizes and shape of the pulsating area. The second ones are expanding and propagating pulsations. This type of pulsation begins with initial brightening of a core and then the patch expands spatially in one or more directions (Scourfield and Parsons, 1971a; Thomas and Stenbaek-Nielsen, 1981). The region of expansion or propagation with horizontal dimensions of tens to hundred kilometers usually retain its shape during several periods. As shown by Scourfield and Parsons (1971a) and Kosch and Scourfield (1992) these types of auroral pulsations can transform from one type to another in a short time interval comparable with a single pulsation period. We deal with the same situation later.

Røyrvic and Davis (1977) studied fine temporal structure of pulsating auroral patches in the frequency range of $2-5 \mathrm{~Hz}$. Sometimes when a patch was not fully 
"switched-off" the fine structure could be seen as rapid intensity modulation both during the maximum and minimum phases of the luminosity. More than $50 \%$ of patches in post-midnight and morning sectors located at equatorward region of auroral zone are modulated. The depth of modulation could reach $20-40 \%$ and it was not constant inside the patch. Sometimes only boundary region is modulated whereas main part is stable. The existence of high-frequency modulation of pulsating auroral patches was also reported by Oguti (1976) and Sandahl (1984). Hansen et al. (1988) found coupled 3 and $1.3 \mathrm{~Hz}$ components in auroral pulsations.

Sandahl et al. (1980) and McEven et al. (1981) carried out in situ electron energy measurements during the rocket launches and showed that the main role in generation of pulsating aurora is played by particles with energies higher than $10 \mathrm{KeV}$.

Electromagnetic ELF and VLF emissions in the frequency range from $100 \mathrm{~Hz}$ to $10 \mathrm{kHz}$ with temporal characteristics similar to pulsating aurora have been observed long time ago. Quasi-periodic ELF and VLF emissions with periods in the range 5-20 s and spiketype intensity variations are reported e.g. in the catalogue (Helliwell, 1965). Chorus elements with periods of fractions of second are often seen. Similar temporal structure often occurs in the pulsating aurora in the form of intensity modulation. Rapid increase of frequency to the end of a spike is typical in the VLF chorus emissions of such type. Narrow-band ELF emissions of hiss type are very often observed simultaneously with VLF emissions outside the plasmapause. Koons (1981) shows, using SCATHA satellite data, that the VLF chorus emissions very often start from the frequencies inside the ELF frequency range and that the chorus emissions could be generated by natural stochastic processes occurring in strongly structured bands of ELF-hiss in the outer magnetosphere.

Tsuruda et al. (1981) report only six cases of peak to peak correlation between chorus elements and pulses of auroral patches during more than one hour of observation. Hansen and Scourfield (1990) have shown that the range of delay times between VLF waves and corresponding optical pulsations is consistent with electron cyclotron source region located near the equatorial plane. Events characterized by VLF risers were presumed to occur in a region of enhanced plasma density commonly observed within the plasma trough by various methods either from the ground by incoherent scatter radar (Vickrey et al., 1980) or by satellites (Muldrew and Vickrey, 1982; Beghin et al., 1984). Manninen et al. (1991) and Zaitsev et al. (1994) also report cases of correlation between auroral and VLF emission pulsations in post-substorm conditions. Novikov et al. (1994) were the first to point out, that a structured VLF emissions are connected with fast moving auroral patches.

Luhmann (1979) proposed the generation of auroral pulsation from atmospheric waves. Stenbaek-Nielsen (1980) pointed that the ionospheric parameters have to be taken into account when studying the auroral pulsation generation. In their interpretations the iono- sphere plays the role of external factor, which can modulate the existing precipitating electron flux. Maehlum and O'Brien (1968) proposed an atmospheric "feed-back" mechanism, which could account for some of the dynamic auroral variations without requiring the auroral source to vary in time. The effect was caused by the changes in pitch angle distribution created by the geomagnetic field deformations caused by the electrojet current system itself. The calculated periodicity of auroral variations between $50-200 \mathrm{~s}$ which is longer than the observed mean values.

Coroniti and Kennel (1970) proposed that the variations in magnetic field strength modulate the rate of pitch-angle diffusion during cyclotron wave-particle interaction at the equatorial plane and this causes pulsating precipitation. This theory was not supported by further satellite and ground-based experiments, which showed that the required hydromagnetic waves coinciding with pulsating events simply did not exist at the equator (Oguti et al., 1986). Scourfield et al. (1972) found that the dimensions of coherently pulsating regions had about a $30-50 \mathrm{~km}$ dimension and separate pulsating patches pulsate independently of each other. It could hardly be so if the modulation were caused by external waves in the magnetosphere. It is now rather generally accepted that pulsating precipitation, causing pulsating auroral patches to appear cannot be related to hydromagnetic waves in the equatorial plane.

The auroral pulsations can be formed by equatorward or poleward motion of luminous auroral forms covering vast regions and propagating as a whole with stable periodicity (Oguti and Watanabe, 1976; Thomas and Stenbaek-Nielsen, 1981). The modulation is not excluded in streaming pulsations (Yamamoto and Oguti, 1982) and fast auroral waves (Cresswell, 1968; Scourfield and Parsons, 1971b) also sometimes cover great regions.

Davidson $(1979,1986,1990)$ and Davidson and Chiu (1986, 1987, 1991) developed the theory based on relaxation oscillator mechanism involving trapped electrons and instabilities generating VLF emissions. The energetic particle flux having pitch-angle anisotropy interact with counter-propagating VLF waves in cyclotron resonance causing particle diffusion into the loss cone. The decrease of anisotropy leads to decrease of growth rate of the waves. The particles are lost when they reach the atmosphere and the loss cone begins to reestablish. The cycle starts again.

Results of simultaneous observations during typical post-breakup period of VLF emissions and auroral pulsation in Sodankylä, Finland $(L=5)$ on February 15,1991 are presented in this work. The data from two selected intervals with 0.5 and 1.0 min durations show strict mutual relation of VLF emissions and auroral pulsation.

The pulsating phenomena are very dynamic and have both spatial and temporal variation even during a single pulse. The separation of these two variations of luminosity is very important when making comparisons with variations of ELF-VLF emissions in order to find the exact time and place of the wave-particle interaction 
causing pulsating precipitation and aurora. The VLF recording contains emissions from rather wide ranging distances and several sources may be active in detectable ranges.

Both auroral and VLF data were handled in digital form and necessary software was developed for auroral image processing (Arinin et al., 1995) and for ELF-VLF signal processing. The software for image processing allows temporal resolution as high as duration of a single TV frame $(0.04 \mathrm{~s})$ and spatial resolution up to $720 \times 512$ pixels. Although only $90 \mathrm{~s}$ of auroral data were analyzed the total number of individual frames was 2250 .

Our explanations for 5-15 s auroral pulsation are based on the theory of the flowing cyclotron maser, which was originally proposed by Trakhtengerts et al. (1985) and Tagirov et al. (1985) and then further developed by Demekhov and Trakhtengerts (1994). It contains inherent assumptions, that the ionosphere is important in formation of auroral pulsation. In this study we propose a new "feed-back" mechanism based of relaxation properties of the ionosphere.

\section{Interpretation of observations}

On 14 February, 1991 the magnetic and auroral activity were moderately high. A clear substorm onset commenced at about 2340 UT. Pulsating aurora was strong during the recovery phase and contained pulsating patches with characteristic periods of 5-10 s, which were expanding and propagating during their brightening. VLF emissions occurred having trains of chorus elements and similar periodicity with auroral pulsations. Chorus elements in frequency range $1.5-2.5 \mathrm{kHz}$ lasted $0.3-0.4 \mathrm{~s}$. Both auroral and VLF activity existed in the $2 \mathrm{~h}$ before to the dawn.

Auroral pulsation were observed by auroral TV camera with $84 \times 105^{\circ}$ field-of-view. The camera was inclined approximately $5^{\circ}$ to the north from the zenith. ELF and VLF data were recorded on the same videotape with TV data. Detailed description of the empirical arrangements is given by Manninen and Turunen (1992). During the analysis the field-of-view was limited to $84 \times 84^{\circ}$. Each TV frame of 0.04 duration was digitized using $256 \times 256$ pixels and 64 brightness levels. One pixel covers $0.55 \times 0.55 \mathrm{~km}$ area at the center of the frame and about $0.7 \times 07 \mathrm{~km}$ at its edges assuming a height level $95 \mathrm{~km}$. Two intervals of observations at 0131:00-0131:30 UT and 0134:35-0135:35 UT were chosen for detailed analysis.

\section{Interval 0131:00-0131:30 UT}

The results of the analysis for the interval 0131:00 0131:30 UT are shown in Fig. 1. Dynamic spectrum of ELF-VLF emissions in the upper panel show quasiperiodic ELF narrow-band emission between 1.2$1.7 \mathrm{kHz}$ and structured spikes of VLF chorus elements at frequencies around 1.5 to $2.5 \mathrm{kHz}$ with $0.3-0.4 \mathrm{~s}$ periodicity from 0131:09 to 0131:18 UT. The maximum frequency of VLF chorus constantly rose during the first half of the interval up to $2.5 \mathrm{kHz}$ at $0131: 14 \mathrm{UT}$. After this it decreased roughly at the same rate to about $1.7 \mathrm{kHz}$ at 0131:18 UT.

Four lower panels show the behavior of auroral luminosity. The location of intensity maximum for each TV frame was calculated as a mean value of intensity in a window with $20 \mathrm{~km}$ diameter. The results are shown in the second and third panels of Fig. 1. The dots show the location of the absolute maximum intensity for each TV frame. They were always in the northeastern sector of the sky.

The luminosity maxima occur in two adjacent regions and the change from one to the other behaves like "onoff switching". One region, named "core A", is located at distances $40-50 \mathrm{~km}$ from the zenith in geomagnetic $\mathrm{N}-\mathrm{S}$ and $25-40 \mathrm{~km}$ in E-W direction. Another region, named "core B" was closer to the zenith at the distances $30-35 \mathrm{~km}$ in geomagnetic N-S direction and $15-20 \mathrm{~km}$ in $\mathrm{E}-\mathrm{W}$ direction. Both cores have dimensions of about $20 \mathrm{~km}$ in N-S and $25 \mathrm{~km}$ in E-W direction. There is an area between the cores where practically no luminosity maxima occur.

The ELF-VLF emissions appeared when the region of the luminosity maxima shifted closer to the zenith (0131:00,:09, :22 UT) into core B region and disappeared when the luminosity maximum shifted to core A region $(0131: 05,: 18,: 25 \mathrm{UT})$.

There were three occurrence of luminosity in core B (0131:00-:05; 0.31:09-:18; 0131:22-:25 UT). The first and the third ones differed by very localized position of the maximum appearance (see the second and the third panels in Fig. 1). Only a few dots appeared outside of the core region. Such situation when only the cores were illuminating was connected with occurrences of narrowband ELF-emissions. The structured VLF emissions during these two intervals are quite weak or absent at all.

Core $\mathrm{B}$ during the second intensification was also very stable and the majority of dots indicating the intensity maxima were located in the same region as in the first and the third intensifications. But, in comparison with these two latter ones, there were many more occurrences of maxima outside the core B in the second intensification, suggesting that an envelope of core B at this instant was not stable. At about 0131:10 UT over about $1-2 \mathrm{~s}$ period the luminosity maxima appeared in southwest direction from the core. The location of the intensity maximum was moving at velocity of about 20 $25 \mathrm{~km} / \mathrm{s}$. At the same time the first discrete VLF emissions appeared starting from the lower ELF range. From 0131.11 UT the luminosity maxima moved to the opposite direction and in 2-3 s reached the extreme position in northeast sector with velocity of about $15 \mathrm{~km} / \mathrm{s}$. Simultaneously the frequency of the chorus elements steadily increased up to 0131:14 UT, when the frequency of chorus spike reached $2.5 \mathrm{kHz}$. For about $1 \mathrm{~s}$ from 0131:17-0131:18 UT the pulsating patch envelope covered the maximum area including both cores A and B (the 5th and the 6th squares in Fig. 2). VLF chorus elements appeared at intervals of fractions of a second. Almost all chorus elements started from the 

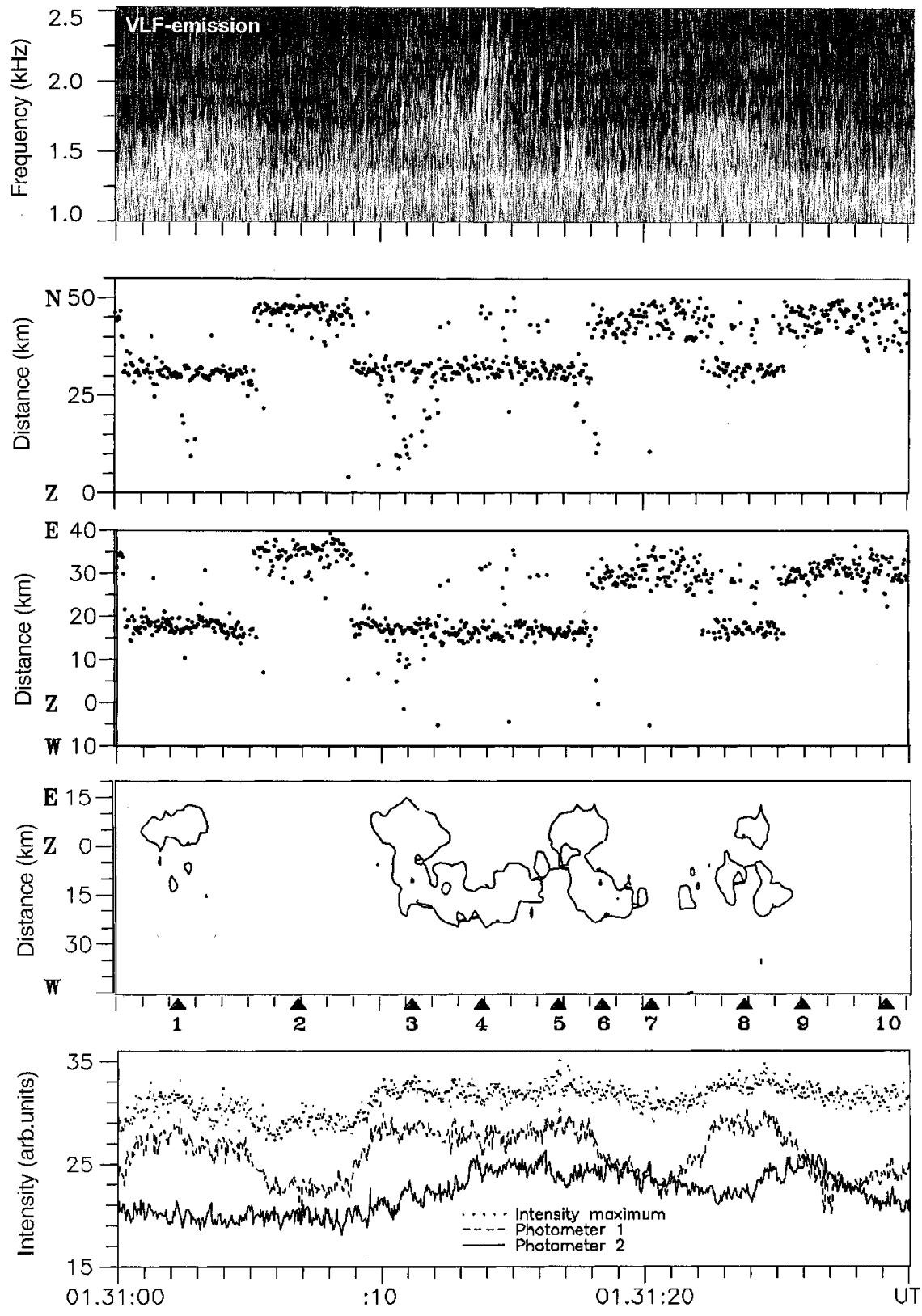

Fig. 1. The results of computer analysis of simultaneous ELF-VLF emission and auroral pulsation data. The uppermost panel shows the dynamic spectrum of the low-frequency emissions. The consequent locations of luminosity maxima for each TV frame in geomagnetic $\mathrm{N}-\mathrm{S}$ and $\mathrm{E}-\mathrm{W}$ directions are shown in the second panel and the third panels, correspondingly. The fourth panel shows the contour of keogram of pulsations (at level of $80 \%$ of maximum luminosity in the interval) along the band marked by the lines in the first square in Fig. 2. $\mathbf{Z}$ means zenith in panels 2 to 4 . Fifth panel presents the variations of intensity maxima (dotted curve) and auroral intensity in two windows shown in Fig. 2 by circles (photometers 1 and 2)
ELF frequency band. Then the upper frequency of chorus elements decreased to 0131:18 UT. Simultaneously a slight southwestward motion occurred in the location of luminosity maximum.

The fourth panel in Fig. 1 shows the keogram of auroral display made approximately in the geographic W-E direction close to the zenith point, along the band between two horizontal lines, shown in the first square in Fig. 2. We present the keogram to show the behavior of the envelope of the core B, which rapidly propagated, at first in geomagnetic westward direction and then back in eastward one, while the core itself was stable. The keogram in Fig. 1 was the most representative to show the motion of the envelope among the set of different keograms tried for this period in different directions across the camera field of view.

The dots outside the core $\mathrm{B}$ center position during the second intensification reveal also the fine structure of the motion of the luminosity maxima. The motion is a sequence of steps and during some steps the intensity of moving illumination exceeds the intensity of the central core. The occurrence frequency of steps is several $\mathrm{Hz}$ and the movement within one step is several kilometers. We will show that the same behavior occurs in the next interval.

Intensity variation of luminosity maxima for the two locations are presented in the fifth panel of Fig. 1. These locations are shown in the second square of Fig. 2 and named here "photometer 1" and "photometer 2". The former was placed in the core region $\mathrm{B}$, the latter was located in the region of luminosity expansion. It is seen that photometer 2 intensity is always lower than photometer 1 intensity. This may explain why the spatial variations of luminosity corresponding to the appearance of VLF emissions is hardly seen in the second and the third panels. The intensity of the core 
region is practically always higher than the expanding envelope. The peak intensity and maximum extent of the expanding part of the luminous area were reached at the instant of the highest VLF chorus frequency (0131:130131:14 UT). The intensity variations measured by
$1 \quad 01.31 .02: 34$ UT

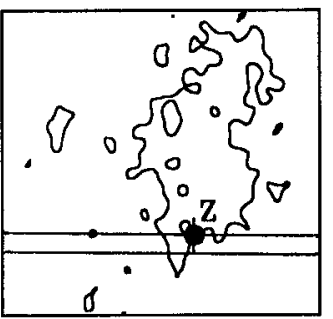

01.31.06:89

2

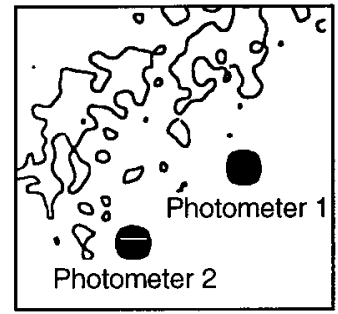

01.31.11:25

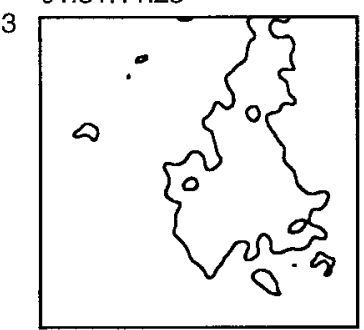

01.31.13:90

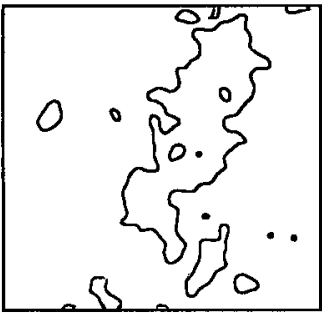

01.31.16:81

5

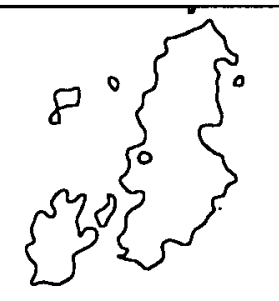

$6 \quad 01.31 .18: 50$
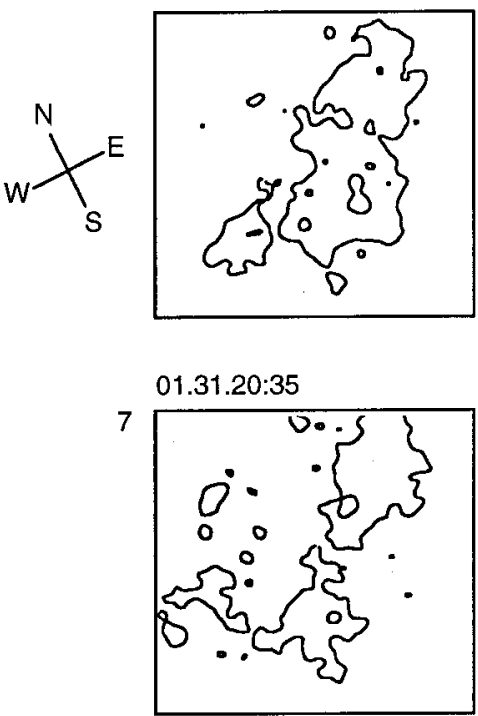

$01.31 .23: 86$

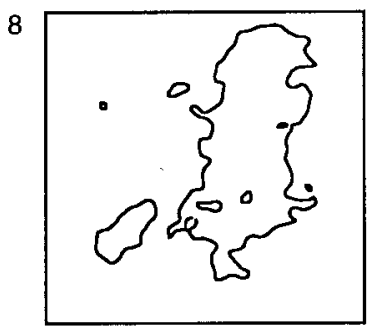

01.31.26:06

9

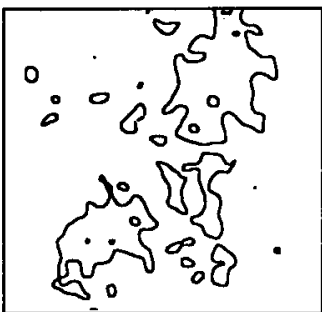

01.31.29:26

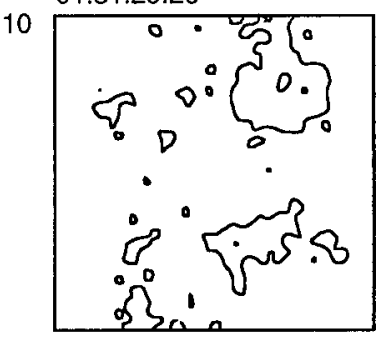

Fig. 2. The contours of the auroral patch for the moments indicated by triangles below the horizontal axis of the fourth panel in Fig. 1. The keogram direction is shown by lines in the first square $Z$ means zenith. Assuming the average altitude about $95 \mathrm{~km}$ the horizontal dimensions of each square are about $130 \times 130 \mathrm{~km}$. The contours as in Fig. 1 photometer 1 correlates fairly well with the lowfrequency emissions. The variations of maximum intensity also correlates with them, but the amplitude is only a few percent above background level.

Further analysis of this interval is presented in Fig. 3. The rectangles show the digitized TV frame. The dots in the upper panel show the distribution of the location of pulsation maxima for the whole interval. The distribu-
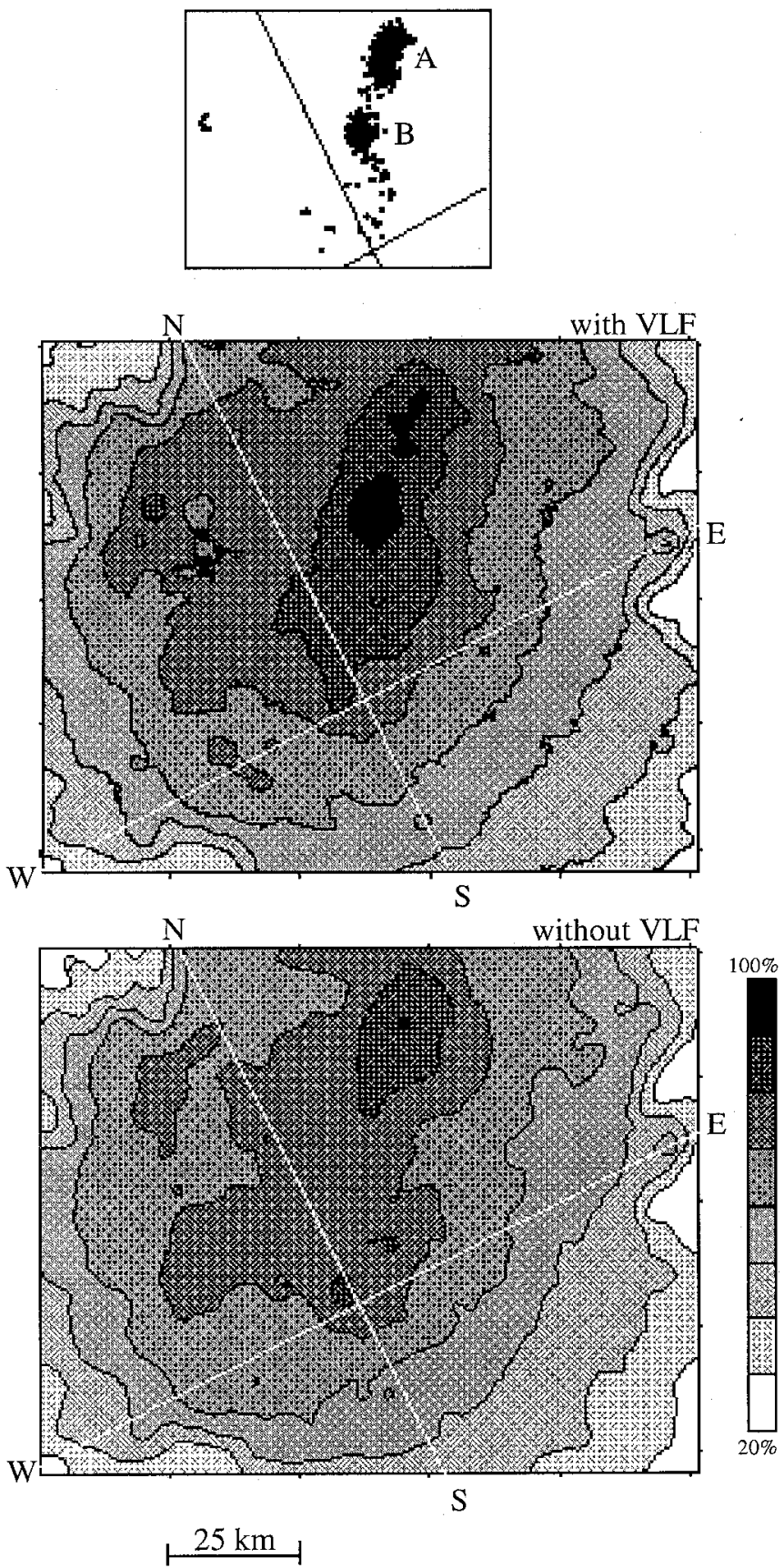

Fig. 3. The uppermost panel presents the spatial distribution of luminosity maxima. The two lower panels represent the distribution of summarized intensity over the whole digitized area for the time intervals when the ELF-VLF emissions occurred (the middle panel) and when they were absent (the lowest panel). The integration times were: 01.31:00-:05; 01.31:09-:18; 01.31:22-:25 UT for the middle panel and 01.31:05-:09; 01.31:18-:22; 01.31:25-:30 UT for the bottom panel 
tion is divided into two clouds corresponding cores A and core B. The cores are separate and the boundary between them is located at a distance of $35 \mathrm{~km}$ from the zenith for the height level of $95 \mathrm{~km}$. The two lower rectangles show the distribution of integrated intensity for the period when ELF-VLF emissions occurred (the middle rectangle) and when they were absent (lower rectangle). This again shows the result mentioned earlier.

The following main experimental facts can be emphasized from the data presented:

1. There were two separate regions named "core A" and "core B" having active pulsating aurora. The regions were separated by about $35 \mathrm{~km}$ in space. Core B intensity was higher than core A intensity by several percent.

2. Auroral pulsation activity in core B was closely connected with the appearance of ELF-VLF emissions, whereas no connection between the lowfrequency emissions was observed in core A.

3. The stable position of core B was connected with noise-like ELF emissions whereas the expansion of luminosity from the core was related to the appearance of VLF chorus risers, which started from the ELF frequency range.

4. The fine structure of the luminosity motion inside the active area was characterized by the stepping position of the luminosity maximum. This happened both in the cores and the envelope. The frequency of the step-like expansion to several kilometers distance was several hertz.

\section{Interval 0134:35-0135:35 UT}

The second analyzed interval is $03 \mathrm{~m} 35 \mathrm{~s}$ later than the one described already. Analysis of the data was identical with the previous interval. Phenomena are very variable but certain common and clearly different features can be recognized.

Dynamic spectrum of ELF-VLF emissions is presented in the upper panel of Fig. 4. The interval contained eight very distinct chorus trains in the frequency range $1.7-2.3 \mathrm{kHz}$. Each of them consisted of 3-6 chorus risers at $0.3-0.4 \mathrm{~s}$ intervals. Most of the intensive riser trains started from intensification of the lower frequency band $(<1.7 \mathrm{kHz})$ which had a hiss character. The keogram in the second panel of Fig. 4. shows that the auroral pulsations propagated rapidly during a single luminosity burst.

The squares in Fig. 5 present the intensity contours of a pulsating patch at different moments. The area used for the keogram is shown by two lines in the first square, (as in Fig. 2 the horizontal dimensions of each square are about $130 \times 130 \mathrm{~km}$ ).

The intensity of the patches rapidly drops when crossing the boundary a few kilometers south of the zenith. Such boundaries are often observed in some types of pulsating aurora, e.g., in "pure" pulsations (Oguti, 1976; Tagirov et al., 1985) and expanding pulsations (Scourfield and Parsons, 1971a). The boundary can retain its shape for several periods of pulsation. One of the possible explanations of these boundaries may be large local gradients of cold plasma inside a tube, where auroral pulsations were generated.

Figure 4. shows, without any doubt, the excellent relationship between the ELF and VLF waves and optical phenomena. There were eight auroral pulses and corresponding chorus trains within this interval. The cycles are shown by thick lines between the panels. The cycle duration varies from 7 to $7.5 \mathrm{~s}$. Although the correlation is fascinatingly clear, it is still difficult to present it by any simple intensity measurements because of dynamic spatial changes.

The third and fourth panels in Fig. 4 show the N-S and E-W components of auroral luminosity maxima propagation. The integrated intensities are shown in Fig. 6 as in Fig. 3. Core A almost disappeared during the second interval and other activity centers appeared and became active. In Fig. 3 the luminosity maxima appeared only few times close to the zenith. On the other hand the shape of the right side of the pulsating pattern seen in Fig. 6 can be seen also in Fig. 3 .

Figure 6 shows that the propagation path of the luminosity maxima formed an arc-like pattern. The luminosity propagated along a steep curved boundary. Those cores, which located at a greater distances from the zenith, show that the curvature of the boundary also existed further westward.

The motion of luminous maxima was more complicated than in the previous case and looked more like a sweeping of brightness moving from one extreme position to another rather than switching on and off. One extreme position approximately coincides with core B location from the previous interval (about $25-35 \mathrm{~km}$ northward and $10-20 \mathrm{~km}$ eastward from the zenith, compare with Fig. 1). Another extreme position can be considered as a new core $\mathrm{C}$, which seems to be a little wider than core B and occupies an area from $5 \mathrm{~km}$ southward to $10 \mathrm{~km}$ northward from the zenith and 5$20 \mathrm{~km}$ westward. The exact times of the contour pictures of the patches in Fig. 5 are shown by arrows in Fig. 4. The mean distance of propagation between cores $\mathrm{B}$ and C was about $40-45 \mathrm{~km}$ in north-southward direction and about $30 \mathrm{~km}$ in east-westward direction. The propagating luminosity moved this distances in a few seconds with a mean propagation velocity between 10 to $12 \mathrm{~km} / \mathrm{s}$.

During this analyzed interval both cores were located in an area of about $30 \mathrm{~km}$ radius. VLF emissions do not coincide with the times when the luminosity is located at the extreme positions, but appear when the luminosity sweeps from core B to core C. Also other cores appeared occasionally in the north-westward direction at distances more than $35 \mathrm{~km}$, but they did not correlate with ELF-VLF activity confirming the result obtained in the first interval for core A.

The fifth panel in Fig. 4 shows the temporal variations of maximum intensity of auroral pulsations. The average intensity constantly increases to the end of the interval. The peak values do not always coincide with 

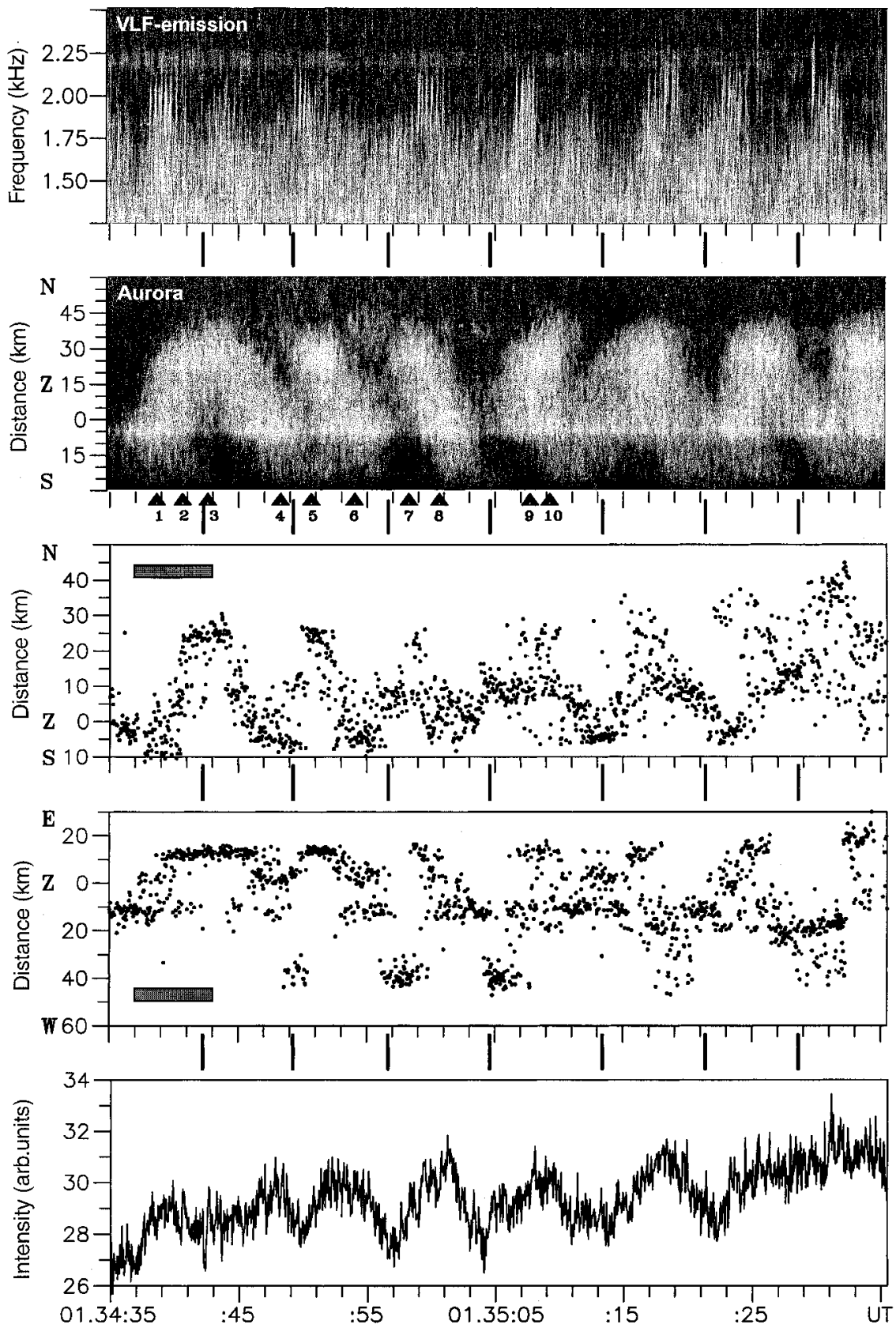

Fig. 4. The results of computer analysis of simultaneous ELF-VLF emission and auroral pulsation data. The uppermost panel shows the dynamic spectrum of the low-frequency emissions. The second panel shows the keogram of auroral pulsations along the direction marked by the lines in the first square in Fig. 5. The consequent locations of luminosity maxima for each TV frame in N-S and E-W directions are shown in the third panel and the fourth panels, correspondingly. $Z$ means zenith in panels 2 to 4. Fifth panel presents the variations of intensity maxima
VLF emission train. The variations of intensity have the same 7.0-7.5 s period and most strong VLF chorus emissions approximately correspond to the maxima of auroral variations (1st, 4th, and 6th). The TV camera measurements were made without any filters and so the time lag effects originating from $0.7 \mathrm{~s}$ lifetime of [OI] $557.7 \mathrm{~nm}$ emission must be remembered, too.

The paths of the moving luminosity maxima to the extreme positions both in N-S and E-W directions form a systematic pattern showing the fine structure of luminosity motion. The luminosity maximum leaped while propagating to extreme positions. Similarly to the first interval the occurrence frequency of the leaps was several hertz and the movement in one leap was several kilometers. It is illustrated in Fig. 7 where we presented the first pulsation from the set in Fig. 4. We enlarged the time scale ten times. The whole time interval was $6 \mathrm{~s}$ from $01.34: 37$ to $01.34: 43 \mathrm{UT}$. It is shown by the black band in the third and fourth panels in Fig. 4. The first panel in Fig. 7 presents the dynamic spectrum of VLF emissions, the motion of luminosity maximum both in $\mathrm{N}-\mathrm{S}$ and E-W directions are shown in the second and third panels, correspondingly. The fourth panel shows the intensity variations of luminosity maximum.

The motion looks like sequential leaps of luminosity maximum to the distances sometimes exceeding $10 \mathrm{~km}$. The thin lines in the second and the third panels presents the bulk motion of luminosity maximum. It is seen that velocity in the central part of the interval in N-S direction was about $7-8 \mathrm{~km} / \mathrm{s}$ and this N-S bulk motion began at about the same time as the first VLF-chorus of the train occurred. Velocity in E-W direction was about 
three times smaller but the distances in the leaps was approximately the same. It seems that when the momentary excursions of maximum luminosity are greater than $20 \mathrm{~km}$ then the maximum luminosity

returns to the previous core thus showing that the intensity of two cores is comparable.

The intensity variations during this interval show that the brightness of intensity maximum slightly increased at the instant of VLF chorus train occurrence and several short-lived peaks of intensity max-

01.34.38:65 UT

1

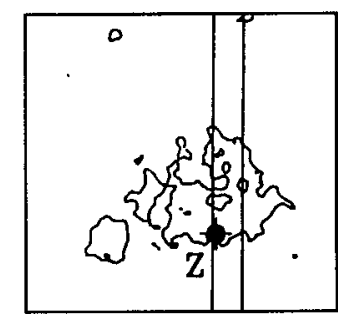

01.34.42:60

3

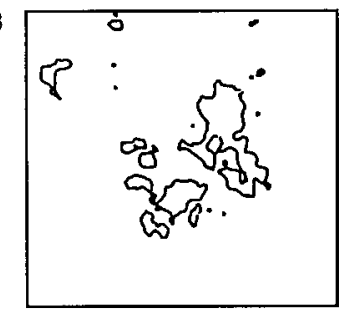

5

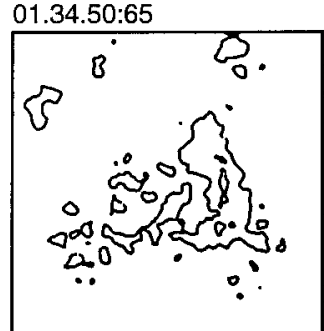

01.34.58:25
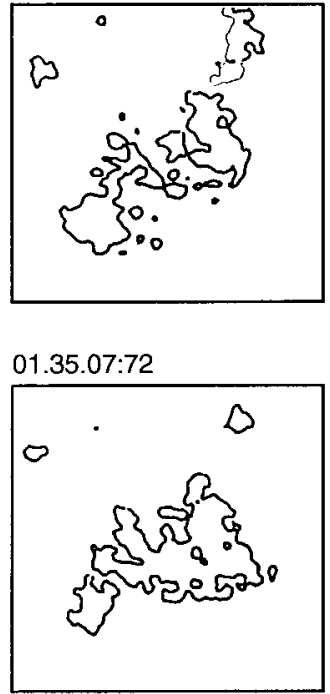

$01.35 .07: 72$

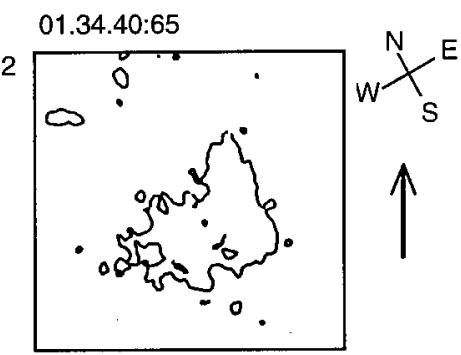

01.34.48:25
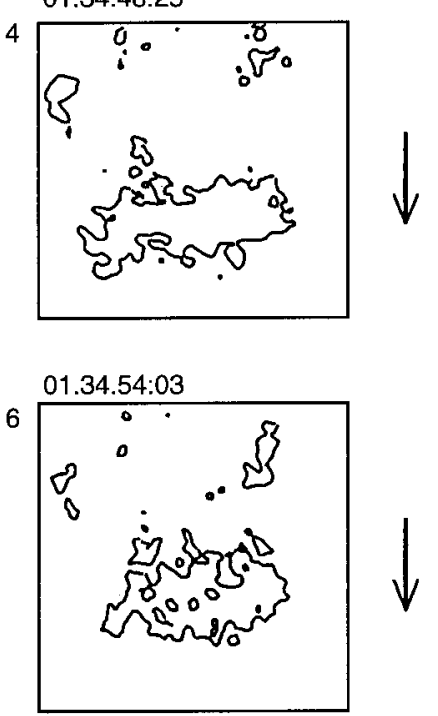

$01.35 .00: 64$

8
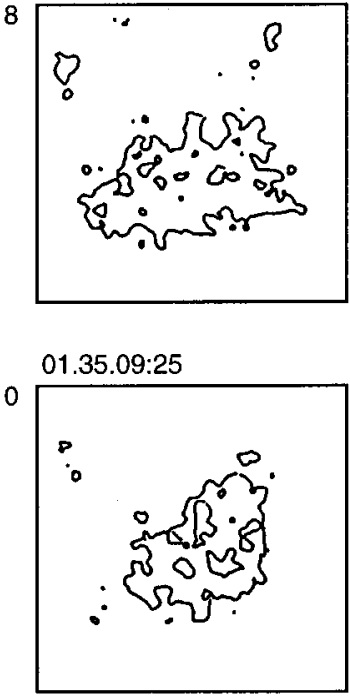

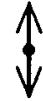

Fig. 5. The contours of the auroral patch for the moments indicated by triangles below the horizontal axis of the second panel in Fig. 4. The keogram direction is shown by lines in the first square. $Z$ means zenith. The contours were drawn at level of $80 \%$ of maximum luminosity in the interval

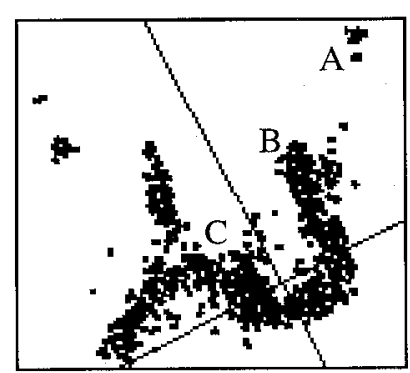

$\mathrm{N}$ with VLF
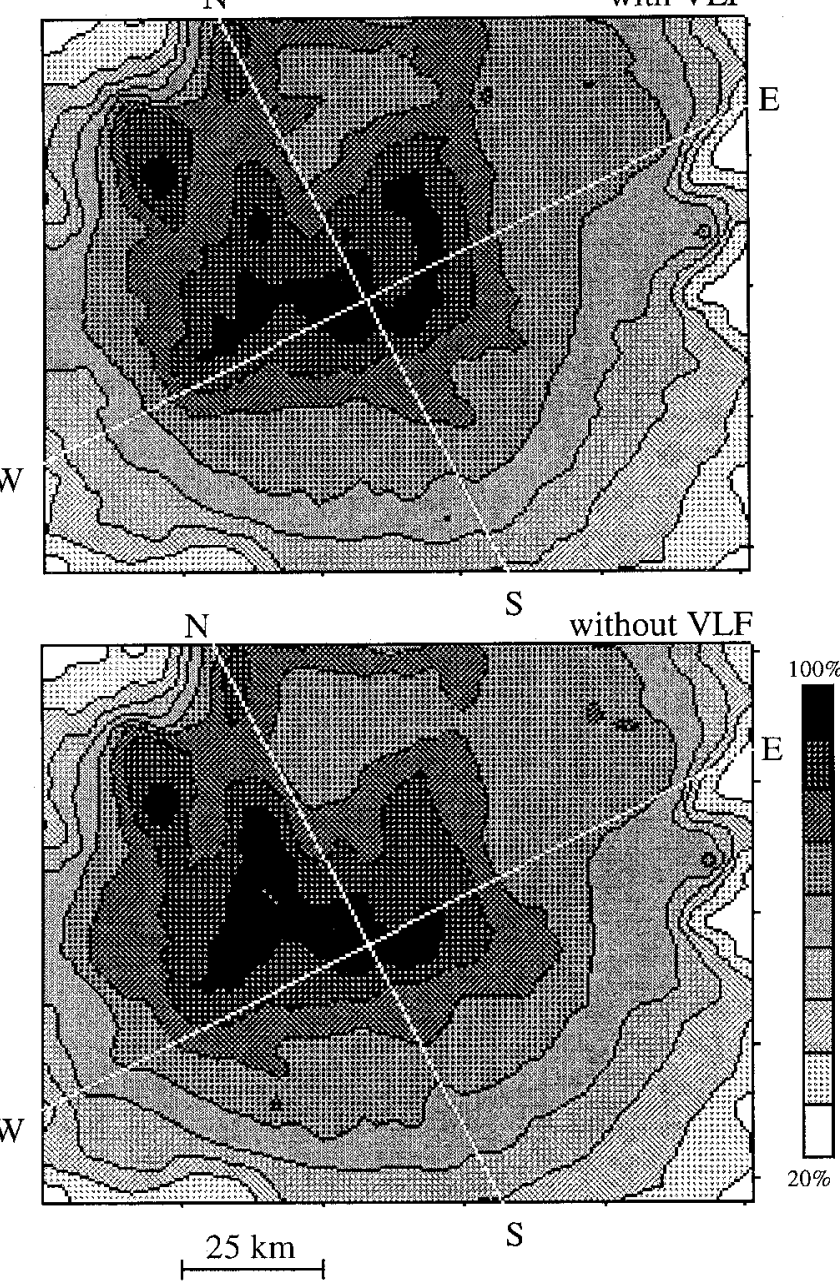

Fig. 6. The uppermost panel presents the spatial distribution of luminosity maxima. The middle panel is auroral intensity distribution for eight intervals when structured VLF chorus trains occurred. The lowest panel is also intensity distribution but for the intervals when VLF emissions were absent. The intervals of integration for middle panel were 8 following: (1) 01.34:37.6-:41.0; (2) 01.34:43.0-:45.2; (3) 01.34:49.4-;53.0; (4) 01.34:56.2-:59.0; (5) 01.35:06.0-:11.0; (6) $01.35: 15.5-: 20.0$; (7) $01.35: 21.4-: 25.0$; (8) 01.35:26.5-:28.5. For the bottom panel the integration was made in between those just mentioned 

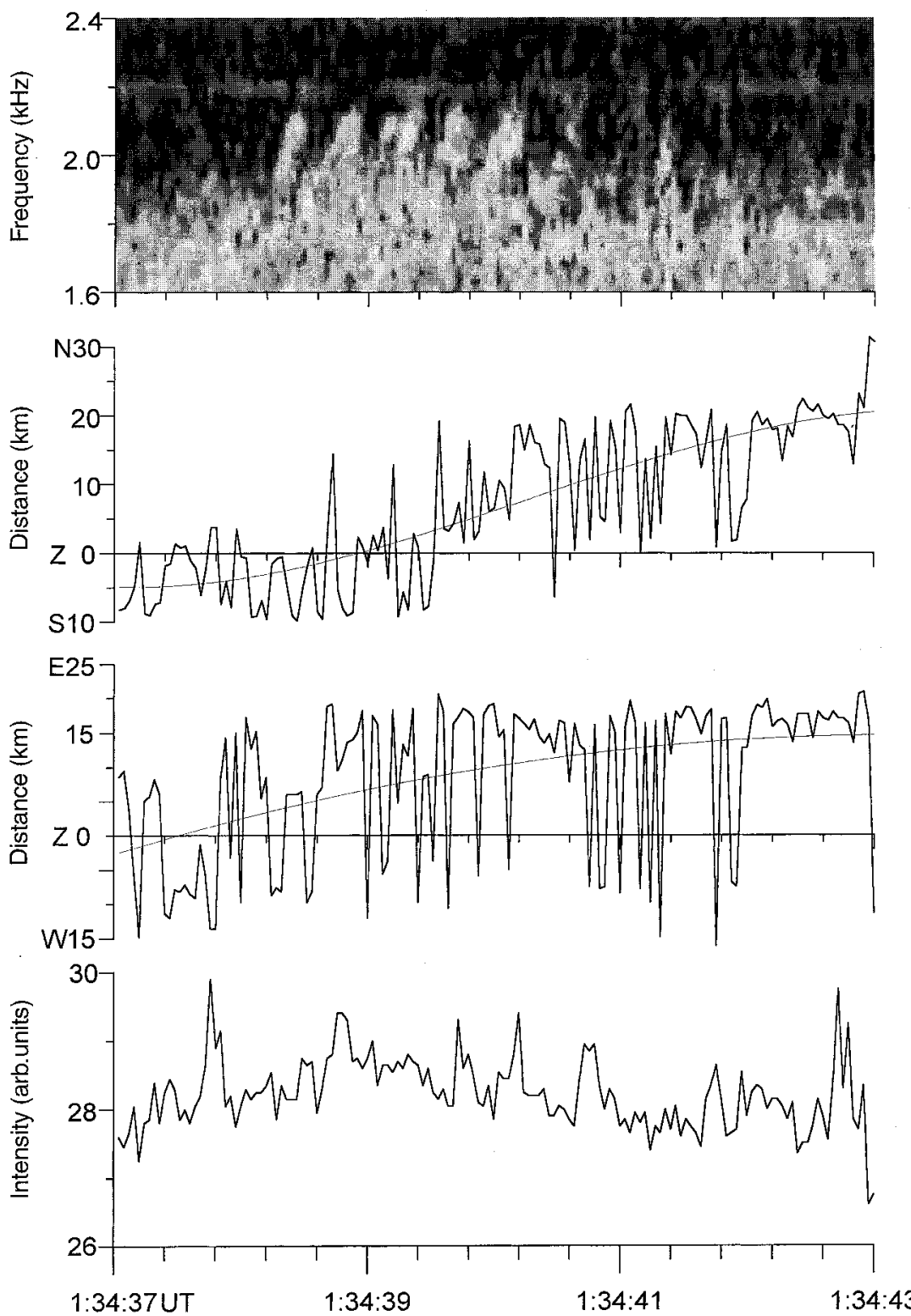

Fig. 7. Detail of the motion and intensity of the single pulsation, the first one from the set in Fig. 4. The whole time interval was $6 \mathrm{~s}$ from $01.34: 37$ to 01.34:43 UT. It is shown by black band in the third panel of Fig. 4. The first panel presents the dynamic spectrum of VLF emissions, the motion of luminosity maximum both in N-S and E-W directions are shown in the second and third panels, correspondingly. Fourth panel shows the intensity variations of luminosity maximum. Thin lines in the second and the third panels presents the averaged motion of luminosity maximum imum $(0.2-0.3 \mathrm{~s})$ took place during the interval. But as mentioned the situation of VLF chorus occurrence with luminosity increase did not repeat for another pulsation (2nd, 3rd and 5th) over the whole $1 \mathrm{~min}$ interval.

The VLF emissions coincide with rapid sweeping motion of the auroral luminosity. There are no obvious peaks in the last two parts of the interval, but the spatial variations of the luminous regions were very strong. The VLF emissions only temporarily coincide with the precise of the maxima of the auroral intensity, but instead they correlated with the rapid propagation of the luminous areas.

These two analyzed periods show, that the pulsating aurora contained moving auroral patches with periods of 7.0-7.5 $\mathrm{s}$ and the velocity of the propagation was 10 $12 \mathrm{~km} / \mathrm{s}$. The propagation took place in the limited region between the two cores at about $50 \mathrm{~km}$ distance from each other. The propagation path was strongly curved.

Trains of structured VLF-risers appeared with the same periodicity as auroral pulsations correlating fairly well with them. The propagation of luminosity was associated with VLF chorus risers $(1.7-2.5 \mathrm{kHz})$. Structured VLF-chorus emissions often started from lower frequency ELF-emissions below $1.7 \mathrm{kHz}$.

The radius of the area where pulsations corresponding to the occurrences of VLF-chorus were observed was about $30 \mathrm{~km}$ slightly northward from the zenith. The other auroral pulsations within the view-of-field did not correlate with the VLF emissions.

The detailed motion of the luminous area inside the active region consists of sequential leaps of the luminosity maximum. The frequency of the leaping motion was several hertz and one individual leap was several kilometers. 


\section{Discussion}

The data analyzed show that the pulsating aurora is very closely connected to low-frequency emissions supporting, in general, the existing theories of cyclotron waveparticle interaction in the magnetosphere as a cause of pulsating auroral phenomena. The most recent reviews of these theories were made by Davidson (1990), Demekhov and Trakhtengerts (1994) and Nemzek et al. (1995).

The theory of the "flowing cyclotron maser" (FCM) proposed by Trakhtengerts and coworkers (Tagirov et al. 1985; Trakhtengerts et al., 1985; Trakhtengerts et al., 1986; Tagirov et al., 1986; Demekhov and Trakhtengerts, 1994) to explain the quantitative experimental results is more advanced than the previous theories. The FCM mechanism involves a few aspects not considered previously together. The first is the existence of a flux tube with an enhanced background cold plasma density playing the role of resonator for ELF-VLF waves. The energetic particles penetrate into the tube while drifting in the magnetic field. The observed finite dimensions of pulsating patches reflect the finite dimensions of the cyclotron instability generation region (Oguti, 1976; Tagirov et al., 1985). The second point is the dynamics of the diffusion rate. The particle drift into the tube is more important than precipitation. The time scale for the new particle population to reach into the flux tube is of the order of $T \sim d / V_{d}$, where $d$ is the width of the tube and $V_{d}$ is the drift velocity of electrons. In this time scale the Alfven maser becomes filled with new energetic electrons having anisotropic pitch-angle distribution. After experiencing the wave particle interactions, the electrons drift out from the tube now having a quasi-isotropic velocity distribution. The possible range of characteristic temporal scales can be very wide.

The pulsating auroral patches are thus produced by the flowing cyclotron maser where the flux tube with increased cold plasma density serves as a resonator. The flux of energetic electrons with anisotropic pitch-angle distribution constantly flowing into the flux tube through its side is the active substance. The flow of the active substance provides constant pumping of the resonator. The cyclotron interaction of the background low-frequency waves with the active substance leads to the process of generation of the waves of ELF-VLF frequency range. The regime of generation has an impulsive character. As a result, from the opposite side of the tube the electrons with isotropic pitch-angle distribution flow out whereas at the butt-ends of the tube emission of ELF-VLF waves and precipitation of resonant electrons causing pulsating auroral patches take place.

In magnetosphere-ionosphere physics the electron density, conductivity and electric field are controlled by ionization-recombination processes. This in turn controls some of the rapid processes occurring in the ionosphere-magnetosphere cavity. The starting point is the continuity equation, which expresses the evolution of interaction between primary flux of precipitating particles and variations of ionization density in the auroral ionosphere:

$\frac{\partial n}{\partial t}=\frac{q}{1+\lambda}-\alpha n^{2}$

where $n$ is the electron density, $\lambda$ is the ratio between the electron and negative ion densities, for the main part of the ionosphere higher than D-region $\lambda \ll 1$, and $\alpha$ is the effective recombination coefficient. This parameter controls the time scales of electron density variations in the ionosphere. Ionization function $q$ is complicated height dependent parameter depending on the density of precipitating particles together with the pitch-angle and energy distributions and atmospheric parameters.

This equation cannot be solved in general way, but the analytical solution can be found if $\alpha=$ const and $q$ is presented in the form $q=q_{0} t^{\chi}$, where $\chi=-2$ or $\chi=$ $4 k(1-2 k)^{-1}$ at $k$ whole, positive or negative (IvanovKholodny and Nikolsky, 1969).

It is easily seen that if $\alpha=$ const, the function $\alpha n^{2}=$ $\varphi(t)=q(t-\Delta t)$ is the solution for the continuity equation if

$\Delta t=(2 \alpha n)^{-1}$

Presenting $q(t-\Delta t)$ as raw, we will get $q(t)-\varphi(t)=$ $\varphi^{\prime} \Delta t=(2 \alpha n)^{-1} 2 \alpha n \cdot n=n$. At the instant the value $\alpha n^{2}$ is equal to the value of function $q$, which it had at the instant $(t-\Delta t)$. The values of $n^{2}$ repeat the variations of $q / \alpha$ with the phase shift increasing with decreasing values of $n$. The constants due to the ionosphere are thus entirely determined by the values of effective recombination coefficient and the electron density.

The effects of pulsating precipitation fluxes to the ionospheric parameters have been studied in several experimental and modeling works. Reid and Phillips (1971) showed that riometer absorption impulses lagged behind X-rays and light emissions at $427.8 \mathrm{~nm}$ by $2.5-$ $5.0 \mathrm{~s}$. A smaller time lag of about 1 second existed between magnetic and riometer pulsations. Campbell (1970) found that the magnetic pulsation lagged behind the luminosity by 0.5 to $3.5 \mathrm{~s}$ with the mean value $1.5 \mathrm{~s}$. In model calculations the response of riometer absorption to the impulse of precipitating particle flux lags by $\sim 2.5 \mathrm{~s}$ comparable with experimental results (Reid and Phillips, 1971).

Johns and Rees (1973) calculated the altitude dependence for the electron density to reach the steady state value using exponential ionization function reaching full value in $4 \mathrm{~s}$. The process is most rapid at the altitude of maximum ionization rate. The modeled aurora reached maximum at $115 \mathrm{~km}$ and the time delay was $5 \mathrm{~s}$. Several factors determine this characteristic time, the most important being the electron density. The authors have chosen the repetition rate of $10 \mathrm{~s}$ for a model calculation of ionization in pulsating aurora. They pointed out that the modulation of the electron density maximizes at the altitude of maximum energy deposition, and there is a 
time lag of a few seconds between the peaks of the ionization function and in the electron density. Phenomena that depend on the latter quantity, e.g., absorption of cosmic radio noise, should have periodicities similar to X-rays or photometric pulsation, but there should be a time lag of a few seconds as shown by Reid and Phillips (1971).

The flux tube acting as resonator has increased cold plasma density. Such plasma filaments, called ELF ducts, were observed by AUREOL-3 satellite in the region of the main ionospheric trough and in the auroral zone (Beghin et al., 1984; Cerisier et al., 1984). The plasma density was increased against the background by 5-20\% and the amplitude of accompanying ELF hiss exceeded the background emissions by one order of magnitude. Typical horizontal sizes of the ducts at Flayer altitudes were $10-50 \mathrm{~km}$, comparable with the typical sizes of pulsating patches. The region of maximum occurrence of the ducts in the morning sector is similar to the appearance of pulsating auroral patches. The authors proposed that these ducts have long lifetime and they can be transported by convection far from the source region. The same convection drift velocity is seen in most cases of pulsating auroral forms (Tagirov et al., 1985; Nakamura and Oguti, 1987). The energetic particles accelerated during the substorm use the slowly drifting ducts for the wave-particle interactions causing pulsating precipitation of the energetic electrons. Pulsation generation, propagation of the waves, particle precipitation and related auroral pulsation at the ends of the duct form a system where both the magnetosphere and ionosphere are coupled together.

The flowing cyclotron maser theory demands that to reach a high efficiency of cyclotron interaction it is necessary that generated waves are stored in the duct volume (Trakhtengerts et al., 1986; Tagirov et al., 1986). Fulfilling the following inequality is sufficient for this:

$\Gamma_{m} \geq|\ln \mathrm{R}|$,

where $\Gamma_{m}$ is maximum amplification of cyclotron waves during one hop travel to equatorial plane and $R$ is the reflection coefficient of the waves at the butt-ends of the duct. There are no studies showing the dependence of $R$ on the ionospheric electron density profile. The greater the electron density the smaller the parameter $R$, because of the absorption in the ionosphere.

Qualitative self-consistent explanation of pulsating auroral patches generation is as follows: the constant flow of energetic particles overtakes the slowly drifting duct and the particle flux limit inside the duct is exceeded at a particular time instant allowing the cyclotron instability to start. The intensity of precipitating flux $(F)$ increases giving rise to enhanced auroral intensity $(I)$. The ionization function $(q)$ is proportional to the auroral intensity without the time-lag, if we consider the observations of $427.8 \mathrm{~nm}$ emission. The ionization function increases but the electron density $(n)$ lags several seconds. Correspondingly the absorption $A$ and reflection coefficient $R$ lag behind the magnetospheric wave and precipitation generation processes by several seconds, but after this time scale the ionospheric electron density begins to reduce the process by attenuating the waves in the ionosphere.

The pulsating process stops when amplification $\Gamma$ and consequently the flux of precipitating electrons $F$ falls to a minimum. Then the relaxation time of the ionosphere governed by recombination processes dictates the time scale. The relaxation time is about twice the build-up time (Johns and Rees, 1973). At the end of the relaxation period electron density $\mathbf{n}$ decreases allowing waves to begin to grow again inside the duct. The whole cycle including build-up and relaxation periods is of the order of $5-15 \mathrm{~s}$, which is very similar to duration of auroral pulsating patches.

Observations of a single patch show that its lifetime might last for several periods i.e., 1.0-3.0 min. Johns and Rees (1973) presented the time history of electron density resulting from ionization function modeled for pulsating aurora with repetition rate of $10 \mathrm{~s}$. The background electron density increases with time and after several periods reaches a level which can totally stop pulsating activity due to increased absorption.

Pulsating patches behave independently except that their bulk motion is similar with typical velocities of several hundreds meters per second. The auroral patch characteristics depend only on internal characteristics of an individual duct i.e., electron density of cold plasma and time depending ionospheric reflection coefficient $R(t)$. These parameters can be different for different patches. The main external factors common to all patches are the flux of energetic particles filling the ducts and the magnetospheric electric field $\mathrm{E}$ forcing the ducts of cold plasma to drift. These parameters can vary slowly in in time and space when compared with the pulsation periods and patch dimensions.

At present there is no theoretical approach to explain the motion of luminous area inside the pulsating auroral patches. Some discussion can be made however, ELFhiss type emissions in the range $1.0-1.7 \mathrm{kHz}$ were very closely correlated with the appearance of the pulsating core. Theoretically the resonant electrons should have the energies between 50 to $90 \mathrm{keV}$. Initial development of cyclotron instability giving rise to chorus elements, luminosity intensity and its expansion or propagation depends on the triggering and growth rate of the waves. This is followed by a decrease controlled by growing absorption of the low-frequency waves. This looks similar to the description of two-stage mechanism of expansion pulsations given by Scourfield and Parsons (1971a), when the core is very little above the background intensity initially and brightens in three consecutive cycles beyond an intensity level at which envelope growth is triggered. However, in several subsequent cycles the core intensity reached at maximum is just below that at which previous triggering occurred, and no envelope appears. A similar situation in our study was seen during the first interval when the triggering was associated with the appearance of VLF risers and slight increase of intensity level above the background. This seems to emphasize that the triggering is closely related to the threshold in precipitating electrons flux intensity or parameters of a duct. 
The theoretical model of the cyclotron maser (Trakhtengerts et al., 1985) contains two pulsation periods, flash interval $T_{f}$ and the pause between flashing $T_{p}$. The flash interval $T_{f}$ has a duration of about $0.3-0.4 \mathrm{~s}$, whereas pause interval is about an order of magnitude longer. The flash interval begins when cyclotron instability switches on and creates a step in the pitch-angle distribution function of electrons at the lowest pitch angles of the distribution. The process of cyclotron instability becomes burst-like and consists of rapid movement of the step in the pitch-angle distribution to higher pitch-angles causing, in turn, an increase of wave frequency and intensity of the waves. The wave-particle interaction develops toward lower energies of electrons around $10-15 \mathrm{keV}$. The flash stops when the pitch-angle distribution became isotropic. Drifting electrons rapidly fill the same cavity, where the precipitation impulse just happened. Decrease of wave energy takes place because of damping by the isotropic particle background, but this process is much slower than the flash time.

Because the energy of the waves is still very high, the wave-particle interaction occupies the adjacent regions with lower densities of cold plasma involving interaction with lower energy electrons from the constantly flowing hot electron flux. This leads to another spike of electron precipitation and chorus riser. This process can repeat several times occupying more and more volume leading to appearance of chorus train and expanding or propagating luminosity. The chorus emissions disappear and luminosity fades when ionospheric ionization under the tube of enhanced cold plasma density begins to limit the process by absorption with the time lag discussed above.

The calculated duration of a flash interval is similar to mean interval between chorus risers in a train usually observed in experiment (see Figs. 1 and 2, upper panels), and the same periodicity is observed in the luminosity of auroral patches (see e.g., Røyrvic and Davis, 1977; Oguti, 1976). This is the $2.5-3.0 \mathrm{~Hz}$ frequency modulation of auroral pulsations. An expanding envelope or motion of the luminosity is thus associated with the slopes of electron density in a duct and related precipitation of lower energy electrons and chorus risers.

\section{Conclusions}

The experimental results described show that the auroral pulsations are closely connected with low-frequency emissions. Two types of low-frequency emissions were observed to be related to optical pulsations, i.e., lowfrequency noise-like ELF-hiss $(<1.7 \mathrm{kHz})$ and higher frequency $(>1.7 \mathrm{kHz})$ structured VLF-chorus emissions. The former was connected to stable pulsating patches whereas the latter correlated with rapidly propagating or expanding auroral pulsations. The auroral situation presented was typical for the recovery phase of a substorm with the pulsating activity of periods 7.0-7.5 s and accompanying ELF-VLF-wave activity.

Most theories of pulsating auroras have so far dealt with the pulsation period and little attention has been given to motion features. These features are, however, closely related with the appearance of structured ELFVLF emissions. Thus motion characteristics, in combination with those of ELF-VLF emissions obtained here, will impose strong restrictions on theoretical models of the causes of pulsations and can be used to test theories proposed so far.

Although there is no measurements of ionospheric parameters presented in this work it is evident that in order to be self-consistent the theories have to take into account the ionosphere under pulsating precipitation as a controlling factor. The relaxation time of the ionosphere is reflected in the pulsation periods and the slowly changing part of the auroral dynamics. A complex study of ionospheric parameters, optical emissions and lowfrequency waves could advance the understanding mechanisms of pulsating phenomena in future experiments.

Acknowledgements. This study has been supported by the INTAS grant 2753. We thank Prof. V. Ye. Trakhtengerts and Prof. D. J. McEven for fruitful discussion of the experimental results. We thank also Mr. Paavo Törmänen who assisted us during the campaign.

Topical Editor K.-H. Glassmeier thanks A. Smith and N. Sato for their help in evaluating this paper.

\section{References}

Arinin, V. A, V. S. Ismagilov, and V. R. Tagirov, Processing of auroral TV images. 19th European Meeting on Atmospheric Studies by Optical Methods, 28 August-1 September 1995, Nurmijarvi, Finland, Program and abstracts. p.F10.

Beghin, C., J. C. Cerisier, J. L. Rauch, J. J. Berthelier, F. Lefeuvre, R. Debrie, O.A. Maltseva, and N. I. Massevitch, Experimental evidence of field-aligned ELF plasma ducts in the ionospheric trough and in the auroral zone, Results of the ARCAD-3 project, Ed. CNES, Toulouse, France, 517-523, 1984.

Campbell, W. H., Rapid auroral luminosity fluctuations and geomagnetic field pulsations, J. Geophys. Res., 75, 6182-6208, 1970.

Cerisier, J. C., J. J. Berthelier, C. Beghin, and D. Roux, Density gradient instability in the high latitude ionosphere. Relation with the electrostatic turbulence and the convection, Results of the ARCAD-3 project, Ed. CNES, Toulouse, France, 509-516, 1984.

Coroniti, F., V. and C. F. Kennel, Electron precipitation pulsations, J. Geophys. Res., 75, 1279-1289, 1970.

Cresswell, G. R., Fast auroral waves, Planet. Space Sci., 16, 14531458, 1968.

Davidson, G. T., Self-modulated VLF-wave-electron interactions in the magnetosphere: a cause of auroral pulsations, J. Geophys. Res., 84, 6517-6523, 1979.

Davidson, G. T., Pitch-angle diffusion in the morning side aurorae, J. Geophys. Res., 91, 4429-4436, 1986.

Davidson, G. T., Pitch-angle diffusion and the origin of temporal and spatial structures in pulsating aurorae, Space Sci. Rev., 53, 45-82, 1990.

Davidson, G. T., and Y. T. Chiu, A closed nonlinear model of waveparticle interaction in the outer trapping and morning side auroral regions, J. Geophys. Res., 91, 13705-13710, 1986.

Davidson, G. T., and Y. T. Chiu, An unusual nonlinear system in the magnetosphere: A possible driver of auroral pulsations, J. Geophys. Res., 96, 19353-19362, 1991.

Davidson, G. T., and Y. T. Chiu, A nonlinear model of waveparticle interactions in the trapped radiation belts: auroral pulsation sources, Geophys. Res. Lett., 14, 1166-1169, 1987. 
Demekhov, A. G. and V. Y. Trakhtengerts, A mechanism of formation of pulsating aurorae, J. Geophys. Res., 99, 58315841,1994

Hansen, H. J., E. Mravlag, and M. W. J. Scourfield, Coupled 3and $1.3 \mathrm{~Hz}$ components in auroral pulsations, J. Geophys. Res., 93,10029-10034, 1988.

Hansen, H. J., and M. W. J. Scourfield, Associated ground-based observations of optical aurorae and discrete whistler waves, J. Geophys. Res., 95, 233-239, 1990.

Helliwell, R. A., Whistlers and related ionospheric phenomena, Stanford University Press, Stanford, California, p.369, 1965.

Inan, U. S., T. F. Bell, and R.A. Helliwell, Non-linear pitch-angle scattering of energetic electrons by coherent VLF waves in the magnetosphere, J. Geophys. Res., 33, 3235-3253, 1978.

Ivanov-Kholodny, G. S., and G. M. Nikolsky, Sun and ionosphere, Nauka Publishing House, Moscow, USSR, p. 455, 1969, (in Russian).

Johns, R. A., and M. H. Rees, Time-dependent studies of the aurora - 1. Ion density and composition, Planet. Space Sci., 21, 537-553, 1973.

Kennel, C. F., Consequences of magnetospheric plasma, Rev. Geophys. Space Phys., 7, 379-419, 1969.

Koons, A. C., The role of hiss in magnetospheric emission, J. Geophys. Res., 86, 6745-6755, 1981.

Kosch M. J., and W.J. Scourfield, Resolution of temporal and spatial ambiguities of intensity variations within pulsating aurorae. J. Geophys. Res., 97, 14877-14883, 1992.

Luhmann, I. R., Auroral pulsations from atmospheric waves, J. Geophys. Res., 84, 4224-4228, 1979.

Maehlum, B. N., and B.J. O'Brien, The mutual effect of precipitating auroral electrons and the auroral electrojet, J. Geophys. Res., 73, 1679-1684, 1968.

Manninen, J., K. Kaila, and T. Turunen, Correlation between pulsating aurora, magnetic pulsations, and VLF chorus observed at Sodankylä, Proc of the 18th Annual Meeting on the Studies of the Upper Atmosphere by Optical Methods, Tromsø, Norway, June 17-21, 29-31, 1991.

Manninen, J., and T. Turunen, Relative motion of whistler channels and auroral forms during an auroral substorm, Proc of 19th Annual European Meeting on Atmospheric Studies by Optical Methods, August 10-14, 1992, Kiruna, Sweden. IRF Scientific Report 209, Ed. by A. Steen, 141-147, 1992.

McEven, D. J., E. Yee, A. W. Yaw, and B.A. Whalen, Energetic particle measurements in pulsating auroras, Can. J. Phys., 59, 1106-1112, 1981

Muldrew, D. B., and J. F. Vickrey, High-latitude $F$ region irregularities observed simultaneously with ISIS 1 and Chatanika radar, J. Geophys. Res., 87, 8263-8272, 1982.

Nakamura, R., and T. Oguti, Drifts of auroral structures and magnetospheric electric fields, J. Geophys.Res., 92, 1124111247, 1987.

Nemzek, R. I., R. Nakamura, D. N. Baker, R. D. Belian, D. J. McCormas, M. F. Thomsen, and T. Yamamoto, The relationship between pulsating auroras observed from the ground and energetic electrons and plasma density measured at synchronous orbit, J. Geophys. Res., 100, 23935-23944, 1995.

Novikov, Y. P., A. A. Mironov, E. E. Titova, T. A. Yahnina, V. R. Tagirov, S. A. Chernouss, V. I. Salin, J. Manninen, and T. Turunen, Spatial-temporal variations of pulsating auroral patches and their relationship with VLF emissions of chorus type, Geomagn. Aeron'o, 34, 32-36, 1994

Oguti, T., Recurrent auroral patterns. J. Geophys. Res., 81, 1782 1786, 1976
Oguti, T., K. Hayashi, T. Yamamoto, J. Ishida, T. Higuchi, and N. Nishitani, Absence of hydromagnetic waves in the magnetospheric equatorial region conjugate with pulsating auroras, J. Geophys. Res., 91, 13711-13715, 1986.

Oguti, T., and T. Watanabe, Quasi-periodic poleward propagation of on-off switching aurora and associated geomagnetic pulsations in the dawn, J. Atmos. Terr. Phys., 38, 543-551, 1976.

Reid, J. S. and J. Phillips, Time lags in the auroral zone ionosphere, Planet. Space Sci., 19, 959-969, 1971.

Rosenberg, T. G., R. A. Helliwell, and J. P. Katsufrakis, Electron precipitation associated with discrete very low frequency emissions, J. Geophys. Res., 76, 8445-8456, 1971

Røyrvic, O., and T. N. Davis, Pulsating aurora: local and global morphology, J. Geophys. Res., 82, 4720-4740, 1977.

Sandahl, I., Pitch-angle scattering and particle precipitation in a pulsating aurora - an experimental study, KGI Report 185, Kiruna, Sweden, 1984, p.161.

Sandahl, I., L. Eliasson, and R. Lundin, Rocket observations of precipitating electrons over a pulsating aurora, Geophys. Res. Lett., 7, 309-310, 1980.

Scourfield, M. W. J., W. F. Innes, and N. R. Parsons, Spatial coherency of pulsating aurora, Planet. Space Sci., 20, 18431848, 1972.

Scourfield, M. W. J., and N. R. Parsons, Pulsating auroral patches exhibiting sudden spatial expansion, J. Geophys. Res., 76, 45184524, 1971a.

Scourfield, M. W. J., and N. R. Parsons, Television imaging of fast auroral waves, Planet. Space Sci., 19, 437-443, 1971 b.

Stenbaek-Nielsen, H. C., Pulsating aurora: the importance of the ionosphere, Geophys. Res. Lett., 7, 353-356, 1980.

Tagirov, V. R., V. Y. Trakhtengerts, and S.A. Chernouss, On the origin of pulsating auroral patches. I. Experimental data, Preprint PGI-85-03-41, p.35, 1985, (in Russian)

Tagirov, V. R., V. Y. Trakhtengerts, and S.A. Chernouss, The origin of pulsating auroral patches, Geomagn. Aeron., 26, 501-505, 1986.

Thomas, R. W., and H. C. Stenbaek-Nielsen, Recurrent propagating auroral forms in pulsating auroras, J. Atmos. Terr. Phys., 43, 243-248, 1981.

Trakhtengerts, V. Y., V. R. Tagirov, and S. A. Chernouss, On the origin of pulsating auroral patches. II. Theory, Preprint PGI-8504-42, p.38, 1985, (in Russian).

Trakhtengerts, V. Y., V. R. Tagirov, and S. A. Chernouss, A circulating cyclotron maser and pulsed VLF emissions, Geomagn. Aeron., 26, 99-106, 1986.

Tsuruda, K., S. Machida, T. Oguti, S. Kokobun, K. Hayashi, T. Kitamura, O. Saka, and T. Watanabe, Correlation between very low frequency chorus and pulsating aurora observed by lowlight-level television at $L=4.4$, Can. J. Phys., 59, 1042-1048, 1981

Vickrey J. F., C. H. Rino, and T. A. Potemra, Chatanika/Triad observations of unstable ionization enhancements in the auroral F-region, Geophys. Res. Lett, 7, 789-792, 1980.

Yamamoto, T. and T. Oguti, Recurrent fast motions of pulsating auroral patches, 1, a case study on optical and quantitative characteristics during a slightly active period, J. Geophys. Res., 87, 7603-7614, 1982.

Zaitsev, D. B., Y. P. Novikov, V. R. Tagirov, and S. A. Chernouss, Diagnostics of spatial-temporal parameters of auroral ionosphere on the basis of optical and low-frequency electromagnetic data, Geomagn. Aeron, 34, 27-31, 1994. 